Proc. Estonian Acad. Sci. Eng., 2005, 11, 2, 91-105

\title{
Comparison between Lagrangian and Eulerian particle simulations coupled with DNS of homogeneous isotropic decaying turbulence
}

\author{
André Kaufmann ${ }^{\mathrm{a}}$, Jerome Helie ${ }^{\mathrm{b}}$, Olivier Simonin ${ }^{\mathrm{b}}$ and Thierry Poinsot ${ }^{\mathrm{b}}$ \\ a CERFACS, 42 Av. G. Coriolis, 31057 Toulouse, France; kaufmann@ cerfacs.fr \\ b IMFT, Av. C. Soula, 31400 Toulouse, France; \{helie, simonin, poinsot $\} @$ imft.fr
}

Received 30 January 2004

\begin{abstract}
A direct numerical simulation (DNS) approach to Eulerian-Eulerian dispersed twophase flow is discussed. The need for a stress term in the momentum transport equation of the dispersed phase is identified and a simple model for this stress term is proposed. Measurements of the dispersed phase quantities such as number density, mesoscopic velocity and stress tensor components from a reference computation, using Lagrangian particle tracking, allows to validate the predictions of the Eulerian-Eulerian computation.
\end{abstract}

Key words: direct numerical simulation, solid particle, turbulent dispersion, continuum approach, preferential concentration.

\section{INTRODUCTION}

Particle-laden flows are of great interest since they occur in a variety of industrial applications such as chemical reactors or internal combustion engines, in which either solid or liquid particles are injected in a gas flow. These twophase flows are characterized by a high level of dynamic coupling and inertia effects, which depend on the particle relaxation time. Commonly used Lagrangian particle tracking techniques are able to handle most of these complex physical processes. However, they are also known to be numerically expensive, as they require a high particle number density to reach a minimum level of accuracy. For unsteady simulations of practical applications of turbulent industrial flows in complex geometries, Lagrangian methods can not be efficiently used today.

As an alternative, a three-dimensional unsteady Eulerian-Eulerian approach is proposed to simulate dispersed two-phase flows. In this approach the dispersed 
phase is treated as the continuous gas phase, through an averaging operator that leads to a system of conservation equations, very similar to those for the gas. The main advantage is therefore that the gas phase algorithm may be used on the dispersed phase with the same numerical accuracy and computational efficiency. However, this averaging operation introduces unclosed terms $\left[{ }^{1}\right]$ in the equations that need to be modelled. These terms are the non-linear term in the transport operator and other terms related to the unresolved part of the particle velocities.

The Eulerian-Eulerian approach has already been successfully applied to Reynolds-averaged simulation (RANS) of turbulent flows. The objective of the present work is to extend this approach to unsteady three-dimensional simulations of complex flows. Long-term objective is to extend this method to an approach comparable to large eddy simulation (LES) as being used in aerodynamics or recently in reactive flows. LES has become a very attractive tool and has proven to be very efficient on inert turbulent flows. Its use for reactive flows is quite recent and its extension to the two-phase flow needs specific developments.

In the present paper, a model for the unclosed non-linear terms of the dispersed phase equations is proposed. This term has a direct impact on the particle momentum transport equation and is crucial in order to capture dynamics and dispersion. It controls the segregation effects that in turn control many other physical processes like mass and heat transfer or particle-particle interactions.

The proposed model is based on an additional stress term in the dispersed phase momentum equation, as described in the first section of the paper. Two different approaches for calculating this stress term may be used. One is tested in the case of homogeneous isotropic decaying turbulence, computed in a quasi DNS mode, and compared to an Eulerian-Lagrangian DNS reference solution. In this case it is possible to derive relations for several integral quantities that are very useful to cross-check the Eulerian and Lagrangian approaches.

\section{THE EULERIAN MODEL}

Eulerian equations for the dispersed phase may be derived by several means. A popular simple way consists of volume filtering of the the separate, local, instantaneous phase equations accounting for the inter-facial jump conditions $\left[{ }^{2}\right]$. Such an averaging approach is very restrictive, because particle sizes and particle distances have to be smaller than the smallest length scale of the turbulence.

A different, not totally equivalent way is the statistical approach in the framework of the kinetic theory. By analogy to the derivation of the Navier-Stokes

equations by kinetic theory [ $\left.{ }^{3}\right]$, a probability density function (PDF) $f_{p}^{(1)}\left(\mathbf{c}_{p} ; \mathbf{x}_{p}, t\right)$ may be defined. This gives the local instantaneous probable number of particles with the given translation velocity $\mathbf{u}_{p}=\mathbf{c}_{p}$. This function obeys a Boltzmann-type kinetic equation, which accounts for momentum exchange with the carrier fluid and for the influence of external forces such as gravity and inter-particle collisions. Transport equations of the first moments (such as particle concentration, mean 
velocity and particle kinetic stress) may be derived directly by averaging from the PDF kinetic equation $\left[{ }^{4}\right]$.

For the sake of simplicity, in this feasibility study interaction forces are limited to drag, considering non-evaporating particles in the absence of gravity. The extension to evaporating flows, gravity force, turbulence corrections in the drag force and other interaction forces is not in conflict with the presented derivation of the Eulerian field equations. In the presented approach, the gas is presumed undisturbed by the dispersed phase. Therefore the passage from one-way to twoway coupling is more delicate.

\subsection{Conservation equations for particle properties}

To derive local instantaneous Eulerian equations in dilute flows (without turbulence modification by the particles), Février et al. $\left[{ }^{5}\right]$ propose to use an averaging over all dispersed-phase realizations, conditioned by one carrier-phase realization. Such an averaging procedure leads to a conditional velocity PDF for the dispersed phase

$$
\tilde{f}_{p}^{(1)}\left(\mathbf{c}_{p} ; \mathbf{x}, t, H_{f}\right)=\left\langle W_{p}^{(1)}\left(\mathbf{c}_{p} ; \mathbf{x}, t\right) \mid H_{f}\right\rangle .
$$

Here $W_{p}^{(1)}$ are the realizations of the position and velocity in time of any given particle $\left[{ }^{6}\right]$ and $H_{f}$ is the unique carrier flow realization. With this definition one may define a local instantaneous particulate velocity field, which is here named "mesoscopic Eulerian particle velocity field". This field is obtained by averaging the conditioned velocity PDF over all particle-flow realizations:

$$
\tilde{u}_{p}\left(\mathbf{x}, t, H_{f}\right)=\frac{1}{\tilde{n}_{p}} \int \mathbf{c}_{p} \tilde{f}_{p}^{(1)}\left(\mathbf{c}_{p} ; \mathbf{x}, t, H_{f}\right) \mathrm{d} \mathbf{c}_{p} .
$$

Here

$$
\tilde{n}_{p}=\int \tilde{f}_{p}^{(1)}\left(\mathbf{c}_{p} ; \mathbf{x}, t, H_{f}\right) \mathrm{d} \mathbf{c}_{p}
$$

is the "mesoscopic" particle-number density and

$$
\langle\tilde{\Phi}\rangle_{p}=\frac{1}{\tilde{n}_{p}^{(1)}} \int \tilde{f}_{p}^{(1)} \Phi \mathrm{d} \mathbf{c}_{p}
$$

stands for any ensemble-averaged quantity.

For simplicity, the dependence of the above variables on $H_{f}$ is not shown explicitly. Application of the conditional-averaging procedure to the kinetic equation, governing the particle PDF, leads directly to the transport equations for the first moments of number density and mesoscopic Eulerian velocity

$$
\frac{\partial}{\partial t} \tilde{n}_{p}+\frac{\partial}{\partial x_{i}} \tilde{n}_{p} \tilde{u}_{p, i}=0
$$




$$
\tilde{n}_{p} \frac{\partial}{\partial t} \tilde{u}_{p, i}+\tilde{n}_{p} \tilde{u}_{p, j} \frac{\partial}{\partial x_{j}} \tilde{u}_{p, i}=-\frac{\tilde{n}_{p}}{\tau_{p}}\left[\tilde{u}_{p, i}-u_{i}\right]-\frac{\partial}{\partial x_{j}} \tilde{n}_{p} \delta \tilde{\sigma}_{p, i j} .
$$

Here $\delta \tilde{\sigma}_{p, i j}$ is the mesoscopic kinetic stress tensor of the particle velocity distribution discussed in Section 2.2. One of the current objectives is to show that this term is non-negligible for inertial particles in the turbulent flow. Due to the very small droplet Reynolds number, measured in the simulation, the particle relaxation time $\tau_{p}$ is defined as the relaxation time for the Stokes drag:

$$
\tau_{p}=\frac{\rho_{p} d^{2}}{18 \mu}
$$

\subsection{The stress tensor of random uncorrelated motion}

The stress term in Eq. (6) arises from an ensemble average of the non-linear term in the transport equation for particle momentum

$$
\begin{gathered}
\tilde{n}_{p} \delta \tilde{\sigma}_{p, i j}=\int\left(c_{p, i}-\tilde{u}_{p, i}\right)\left(c_{p, j}-\tilde{u}_{p, j}\right) \tilde{f}_{p}^{(1)}\left(\mathbf{c}_{p} ; \mathbf{x}, t, H_{f}\right) \mathrm{d} \mathbf{c}_{p}, \\
\tilde{n}_{p} \delta \tilde{\sigma}_{p, i j}=\tilde{n}_{p}\left\langle\delta u_{p, i} \delta u_{p, j} \mid H_{f}\right\rangle_{p},
\end{gathered}
$$

and contains the uncorrelated part of the particle motion. The uncorrelated part of the particle velocity is here referred to as random uncorrelated motion (RUM) ${ }^{1}$. When the Euler or Navier-Stokes equations are derived from kinetic gas theory, the trace of $\left\langle\delta u_{p, i} \delta u_{p, j}\right\rangle_{p}$ is interpreted as temperature (ignoring the Boltzmann constant and molecular mass) and related to pressure by an equation of state. In the case of the Euler or Navier-Stokes equations, the temperature is defined as the uncorrelated part of the kinetic energy. Here the uncorrelated part of the particulate kinetic energy is defined as

$$
\delta \tilde{\theta}_{p}=\frac{1}{2}\left\langle\delta u_{p, i} \delta u_{p, i} \mid H_{f}\right\rangle_{p}
$$

By analogy to the Euler or Navier-Stokes equations, a random uncorrelated motion pressure (RUMP) may be defined by the product of uncorrelated kinetic energy and particle number density as

$$
P_{\mathrm{RUM}}=\tilde{n}_{p} \frac{2}{3} \delta \tilde{\theta}_{p}
$$

1 Random uncorrelated motion (RUM) has been referred to as quasi Brownian motion $(Q B M)$ in previous publications $\left[{ }^{1,5,7}\right]$. We agree that the expression quasi Brownian is misleading since the physical interpretation of the uncorrelated motion is not of Brownian nature. 
When the particle number distribution becomes non-uniform, as in the case of a compressible gas, this pressure term tends to homogenize the particle number density.

The non-diagonal elements of the stress tensor can be identified, by analogy to the Navier-Stokes equations, as a viscous term due to shear. The diagonal part of the stress tensor is then proportional to one third of the trace of the tensor and an eventual deviation, such that $\tilde{n}_{p} \delta \tilde{\sigma}_{p, i j}=P_{\mathrm{RUM}} \delta_{i j}-\delta \tilde{\tau}_{p, i j}$. The momentumtransport equation (6) becomes

$$
\tilde{n}_{p} \frac{\partial}{\partial t} \tilde{u}_{p, i}+\tilde{n}_{p} \tilde{u}_{p, j} \frac{\partial}{\partial x_{j}} \tilde{u}_{p, i}=-\frac{\tilde{n}_{p}}{\tau_{p}}\left[\tilde{u}_{p, i}-\tilde{u}_{f, i}\right]-\frac{\partial}{\partial x_{i}} P_{\mathrm{RUM}}+\frac{\partial}{\partial x_{j}} \delta \tilde{\tau}_{p, i j} .
$$

By analogy to the derivation of the Navier-Stokes equations from kinetic gas theory, the stress term can be related to the gradients of the first moments, based on the Onsager relations $\left[{ }^{8}\right]$. Making some assumptions on symmetry and isotropy, the stress term can be modelled as

$$
\tilde{\tau}_{p, i j}=\mu_{\mathrm{RUM}}\left(\frac{\partial \tilde{u}_{p, i}}{\partial x_{j}}+\frac{\partial \tilde{u}_{p, j}}{\partial x_{i}}-\frac{2}{3} \frac{\partial \tilde{u}_{p, k}}{\partial x_{k}} \delta_{i j}\right) .
$$

The dynamic viscosity, related to RUM, can be estimated by $\mu_{\mathrm{RUM}}=$ $1 / 3 \tilde{n}_{p} \tau_{p} \delta \tilde{\theta}_{p}\left[{ }^{1}\right]$, where $\tau_{p}$ is the relaxation time related to Stokes drag. This expression can be obtained using the transport equation for the complete stresses $\left\langle\delta u_{p, i} \delta u_{p, j}\right\rangle$ and supposing isotropic behaviour in shear flow $\left[{ }^{3}\right]$.

Preliminary tests with the closure model, given in Eq. (13), failed since excessive segregation prevented the completion of the numerical simulation. There are two possible origins of this difficulty: the chosen spatial resolution is insufficient to resolve the physics or the closure model does not describe the proper physics. Supposing that the numerical resolution of the model is insufficient, a subgrid model, acting on the compressible component of the velocity, is chosen. This subgrid model has the form of a bulk viscous term $\xi_{\mathrm{SGS}} \partial \tilde{u}_{k} / \partial x_{k} \delta_{i j}$, which is added to the shear viscosity term $\tilde{\tau}_{p, i j}$ in Eq. (13). The subgrid bulk viscosity is mesh-size dependent $\left(\xi_{\mathrm{SGS}} \propto \tilde{n}_{p}(\Delta x)^{2}\left|\partial \tilde{u}_{k} / \partial x_{k}\right|\right)$. In homogeneous turbulence, the spatial average of this bulk viscous term is zero; still it acts locally and leads to a smoothing of the number density field. Computations are performed with this heuristically introduced bulk viscosity. The closure model (13) requires the knowledge of $\delta \tilde{\theta}_{p}$, which is developed in the next section.

\subsection{The equation for random uncorrelated kinetic energy (RUE)}

To calculate the RUE, two approaches are presented; the first one assumes a quasi-isentropic behaviour of the dispersed phase, leading to an algebraic expression for $\delta \tilde{\theta}_{p}$

$$
\delta \tilde{\theta}_{p}=A \tilde{n}_{p}^{2 / 3}
$$


where $A$ is the residual mean kinetic energy of the particles, averaged over the computational domain, weighed by moments of the particle distribution. In order to define $A$, it is necessary to introduce some definitions. Spatial averages (over the computational domain) are defined as $\{\phi\}=1 / V \int_{V} \phi \mathrm{d} V$. Particle-pondered averages are defined as $\{\phi\}_{p}=\left\{\tilde{n}_{p} \phi\right\} /\left\{\tilde{n}_{p}\right\}$. This allows to define $A$ as $A=\left\{\tilde{n}_{p}\right\} \delta q_{p}^{2} /\left\{\tilde{n}_{p}^{5 / 3}\right\}$. The mean residual particle kinetic energy is defined as $\delta q_{p}^{2}=\left\{\delta \tilde{\theta}_{p}\right\}_{p}$. Equation (14) relates therefore the kinetic energy of the local residual particle to the mean kinetic energy of the residual particle. It can be obtained using the conservation equation for number density (Eq. (5)) and the lowest order conservation equation for RUE in the Chapman-Enskog expansion [ $\left.{ }^{3}\right]$

$$
\frac{\partial}{\partial t} \tilde{n}_{p} \delta \tilde{\theta}_{p}+\frac{\partial}{\partial x_{j}} \tilde{n}_{p} \tilde{u}_{p, j} \delta \tilde{\theta}_{p}=-\frac{2}{3} \tilde{n}_{p} \delta \tilde{\theta}_{p} \frac{\partial \tilde{u}_{p, j}}{\partial x_{j}} .
$$

A conservation equation for $\tilde{n}_{p}^{-2 / 3} \delta \tilde{\theta}_{p}$ can be obtained combining Eq. (5), multiplied by $-\frac{2}{3} \tilde{n}_{p}^{-5 / 3} \delta \tilde{\theta}_{p}$ and Eq. (15), multiplied by $\tilde{n}_{p}^{-2 / 3}$. With the above assumptions, $\tilde{n}_{p}^{-2 / 3} \tilde{\theta}_{p}$ is a conserved quantity and locally $\tilde{\theta}_{p}$ should be proportional to $\tilde{n}_{p}^{2 / 3}$. This property was tested using the results of Lagrangian particle tracking in homogeneous isotropic turbulence. Figure 1 shows the conditional average of RUE as a function over particle number density at different times of the simulation

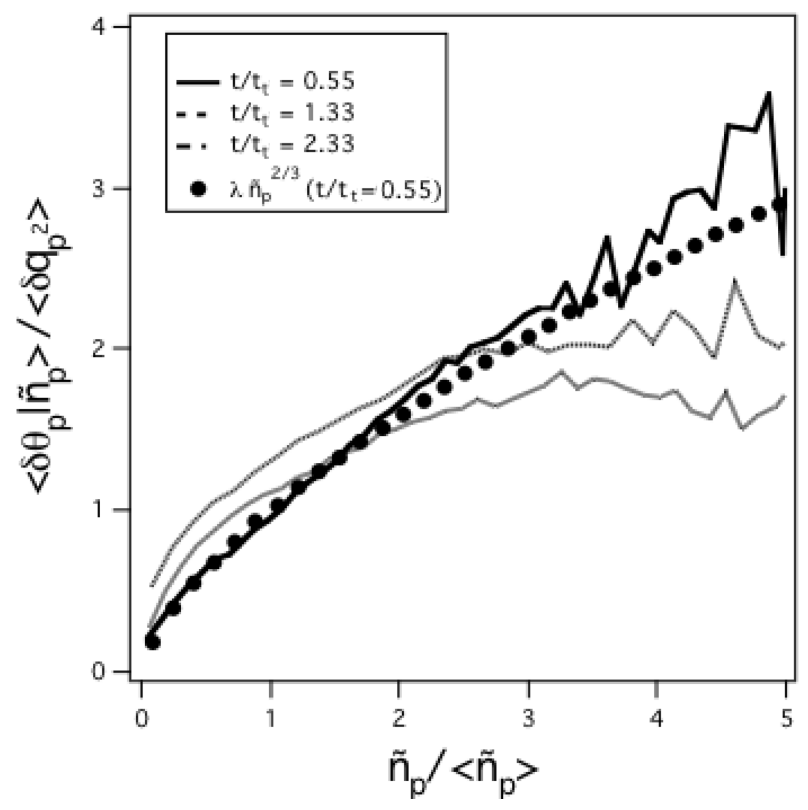

Fig. 1. Conditional average of RUM kinetic energy vs the number density from Lagrangian simulation at different times of the simulation. 
and shows the validity of this isentropic approximation. Lagrangian simulations, performed by Février $\left[{ }^{7}\right]$ in stationary homogeneous isotropic turbulence, suggest that the mean residual kinetic energy $\delta q_{p}^{2}$ depends on the resolved dispersed phase kinetic energy $\tilde{q}_{p}^{2}=1 / 2\left\{\tilde{u}_{p, k} \tilde{u}_{p, k}\right\}_{p}$, on the fluid-particle correlation $q_{f p}=$ $\left\{\tilde{u}_{p, k} u_{k}\right\}_{p}$, where $u_{k}$ is the carrier phase velocity, and on the carrier phase kinetic energy, pondered by the particle presence $q_{f @ p}^{2}=1 / 2\left\{u_{k} u_{k}\right\}_{p}$. This expression is given as

$$
\delta q_{p}^{2}=\tilde{q}_{p}^{2}\left(\frac{4 \tilde{q}_{p}^{2} q_{f}^{2} @ p}{q_{f p}^{2}}-1\right) .
$$

The second approach uses the full transport equation for random uncorrelated kinetic energy $\left[{ }^{1}\right]$. This equation is an equivalent to the internal energy equation of the Navier-Stokes equations:

$$
\begin{aligned}
& \frac{\partial}{\partial t} \tilde{n}_{p} \delta \tilde{\theta}_{p}+\frac{\partial}{\partial x_{j}} \tilde{n}_{p} \tilde{u}_{p, j} \delta \tilde{\theta}_{p}=-2 \frac{\tilde{n}_{p}}{\tau_{p}} \delta \tilde{\theta}_{p} \\
& \quad-\left[P_{\mathrm{RUM}} \delta_{i j}-\xi_{\mathrm{SGS}} \frac{\partial \tilde{u}_{p, k}}{\partial x_{k}} \delta_{i j}-\mu_{\mathrm{RUM}}\left(\frac{\partial \tilde{u}_{p, i}}{\partial x_{j}}+\frac{\partial \tilde{u}_{p, j}}{\partial x_{i}}-\frac{2}{3} \frac{\partial \tilde{u}_{p, k}}{\partial x_{k}} \delta_{i j}\right)\right] \frac{\partial \tilde{u}_{p, i}}{\partial x_{j}} \\
& \quad+\frac{\partial}{\partial x_{j}}\left[k_{\mathrm{RUM}} \frac{\partial}{\partial x_{j}} \delta \tilde{\theta}_{p}\right] .
\end{aligned}
$$

The third-order correlation is modelled as a diffusive flux like thermal diffusion in the Navier-Stokes equations. The diffusivity constant for RUE is estimated as $k_{\mathrm{RUM}}=5 / 3 \tilde{n}_{p} \tau_{p} \delta \tilde{\theta}_{p}$. This is the equivalent of the Fick's law for the heat flux in the Navier-Stokes equations.

\section{DESCRIPTION OF THE NUMERICAL TEST}

Homogeneous isotropic turbulence is one of the classical cases, where the dynamics and dispersion of particle laden flows can be studied. This has been done extensively using the Lagrangian formalism and encouraging results and insight have been obtained with such methods. Comparison of Lagrangian particle tracking in decreasing homogeneous isotropic turbulence $\left[{ }^{9}\right]$ with experimental measurements of particle dispersion in grid generated turbulence $\left[{ }^{10}\right]$ show that essential features of the particle dynamics can be captured. Preliminary computations with a simplified Eulerian formalism of this test case gave encouraging results $\left[{ }^{11}\right]$. In the case of tracer particles (small Stokes number limit) Eulerian methods are well suited to describe the dynamics $\left[{ }^{2}\right]$. With increasing Stokes number, the particle velocities become decorrelated with the gaseous carrier-phase velocity. Inertia effects become important and segregation 
occurs for Stokes numbers about unity. The subject of the study is therefore not only the development of an adequate numerical tool but to show the validity of the Eulerian approach for Stokes numbers from the tracer limit $(S t \rightarrow 0)$ to unity.

\subsection{Initialization of the test case: the gaseous velocity field}

The gaseous carrier phase is initialized by a divergence-free velocity field, obeying a Passot-Pouquet spectrum for the kinetic energy:

$$
E(k)=C\left(\frac{k}{k_{e}}\right) e^{-2\left(k / k_{e}\right)^{2}} .
$$

Here $k$ is the wavelength and $k_{e}$ corresponds to the most energetic wavelength. The initial gaseous solution is then numerically advanced over a characteristic time scale of the turbulence in order to establish a physical spectrum and velocity field that is the solution of the Navier-Stokes equations.

The Reynolds number $R e=u^{\prime} l / \nu$, based on the integral length scale $l$ and $u^{\prime}$ is 18 . The spatial resolutions of the Eulerian simulations are $64^{3}$ and $128^{3}$ equidistant nodes. After a non-dimensional time of $t=4.23 \mathrm{~s}$, the Reynolds number of the carrier phase is 14 . This flow field is taken as the initial flow field for the carrier phase for all test cases.

Length scales and velocities were made dimensionless by using the length scale $l=0.001 \mathrm{~m}$ and velocity scale $c=347 \mathrm{~m} / \mathrm{s}$. The corresponding time scale is $t=2.8818 \times 10^{-6} \mathrm{~s}$.

\subsection{Initialization of the test case: the dispersed phase velocity field}

Several options exist to initialize the dispersed phase, although there does not exist a natural way for that. Here particles are considered equally distributed throughout the computational domain when computation of the dispersed phase is started. Special attention is taken for the initialization of the dispersed phase velocity. Three principal possibilities exist:

1) dispersed phase velocity equals carrier phase velocity,

2) dispersed phase velocity equals zero,

3 ) dispersed phase velocity is partially correlated to the carrier velocity.

In the case of relaxation times, small compared to the characteristic time scales of the carrier phase, the particles velocity field is close to the carrier phase velocity field. In this case it is physical to initialize the dispersed phase velocity field with the gaseous velocity field and to initialize the RUE field with a value close to zero. The RUE field can not be initialized with zero since shear viscosity and RUE flux coefficients are directly linear in RUE. Here only the first initialization method is considered. Furthermore, simulations are made in the unfavorable case of the Stokes number $\left(S t=\tau_{p} / T_{f}\right)$ close to unity, leading to maximal segregation effects; here $T_{f}=\lambda / u^{\prime}$ is the turnover time and $\lambda$ is the integral length scale of the carrier phase. 


\section{COMPUTATION OF THE LAGRANGIAN REFERENCE SOLUTION}

The Lagrangian particle tracking method is a well understood tool for the numerical investigation of particle-laden turbulence. In the case of Stokes drag, the particle coordinate and velocities are advanced in time with the following set of differential equations:

$$
\begin{gathered}
\frac{\partial}{\partial t} X_{i}^{(k)}=V_{i}^{(k)} \\
\frac{\partial}{\partial t} V_{i}^{(k)}=\frac{1}{\tau_{p}}\left(u_{i}\left(X_{i}^{(k)}, t\right)-V_{i}^{(k)}\right) .
\end{gathered}
$$

In realistic applications particle numbers are so large that it is not possible to track all the particles individually and particles are advanced as "numerical" particles that are supposed to represent a large number of "physical" particles. In order not to bias the result by such a procedure, here all particles are computed individually. Special care is taken to evaluate the gaseous velocity $u_{i}$ at the particle location for the drag force by using high-order interpolation methods $\left[{ }^{12}\right]$.

The spatial resolution of the gaseous phase is $64^{3}$ and an average of 40 particles are computed per gaseous node. This corresponds to a total of 10.48 million individual particles. This high particle number ensures convergence when averaged fields are computed from the discrete particle distribution. The averaged fields are sensitive to the numerical procedure used. With the high number of particles used, the error can be shown to be smaller than $3 \%$ by comparing different averaging methods. The correlated velocity of the particles is calculated by a filtering procedure, projecting the correlated velocities on the numerical grid of the carrier phase. Initially, as for the Eulerian simulation, particle velocities are either initialized with the gaseous velocities or zero.

\section{DYNAMICS OF DISPERSED TWO-PHASE FLOWS IN HOMOGENEOUS ISOTROPIC TURBULENCE}

Integral properties of decaying homogeneous isotropic turbulence can be described by a simple set of ordinary differential equations of the integral kinetic energy and the dissipation of kinetic energy

$$
q_{f}^{2}=\frac{1}{2}\left\{u_{k} u_{k}\right\}, \quad \varepsilon=\frac{\nu}{2}\left\{\frac{\partial u_{i}}{\partial x_{j}} \frac{\partial u_{i}}{\partial x_{j}}\right\}
$$

where

$$
\frac{\partial}{\partial t} q_{f}^{2}=-\varepsilon, \quad \frac{\partial}{\partial t} \varepsilon=-C_{2} \frac{\varepsilon^{2}}{q_{f}^{2}},
$$

and $\nu$ is the kinematic viscosity. 
These equations can be obtained from the Navier-Stokes equations with some assumptions on the properties of the flow $\left[{ }^{13}\right]$, satisfied in incompressible decaying homogeneous turbulence. Using the Lagrangian equations of particle transport with Stokes drag (19), (20), a corresponding set of ordinary differential equations for the fluid-particle correlation and the particle kinetic energy

$$
q_{f p}=\frac{1}{N} \sum_{k=1}^{N} u_{i}\left(X_{i}^{(k)}, t\right) V_{i}^{(k)}, \quad q_{p}^{2}=\frac{1}{N} \sum_{k=1}^{N} V_{i}^{(k)} V_{i}^{(k)}
$$

can be obtained for the dispersed phase $\left[{ }^{14}\right]$ :

$$
\begin{gathered}
\frac{\partial}{\partial t} q_{f p}=-\frac{q_{f p}}{\tau_{f p}^{t}}-\frac{1}{\tau_{p}}\left[q_{f p}-2 q_{f @ p}^{2}\right], \\
\frac{\partial}{\partial t} q_{p}^{2}=-\frac{1}{\tau_{p}}\left[2 q_{p}^{2}-q_{f p}\right] .
\end{gathered}
$$

Here $\tau_{f p}^{t}$ corresponds to the turbulence time scale that governs the dissipation of fluid-particle correlation. Equation (24) can then be used to calculate this dissipative time scale a posteriori:

$$
\tau_{f p}^{t}=-\frac{q_{f p}}{\frac{\partial}{\partial t} q_{f p}+\frac{1}{\tau_{p}}\left[q_{f p}-2 q_{f @ p}^{2}\right]} .
$$

The time scale for the dissipation of the carrier phase $\tau^{+}=q_{f}^{2} / \varepsilon$ and the relaxation time of the particles $\tau_{p}$ can then be compared to the dissipative time scale of fluid-particle correlation. For comparison, the residual kinetic energy is computed from the square of the difference between the filtered Lagrangian velocity and the individual particle velocity.

\section{RESULTS AND DISCUSSION}

Numerical computations are performed using the initialization procedure, described in Section 3. The temporal development of the integral values for kinetic energies and fluid-particle correlation are presented in Fig. 2. Numerical results of the Eulerian computation (lines) are compared to the numerical results of the Lagrangian reference computation (symbols). In the Lagrangian computation the correlated kinetic energy is calculated using the correlated velocity. The RUE is then calculated as the difference between the correlated kinetic energy and the total kinetic energy, obtained as the sum of kinetic energies of all the particles. In the Eulerian computation, the total kinetic energy of the particles $q_{p}^{2}$ is obtained from the sum of the resolved kinetic energy $\tilde{q}_{p}^{2}$ and the RUE $\delta q_{p}^{2}$. The open triangles give the RUE, estimated by the values from the equilibrium formula (16). Initially RUE 


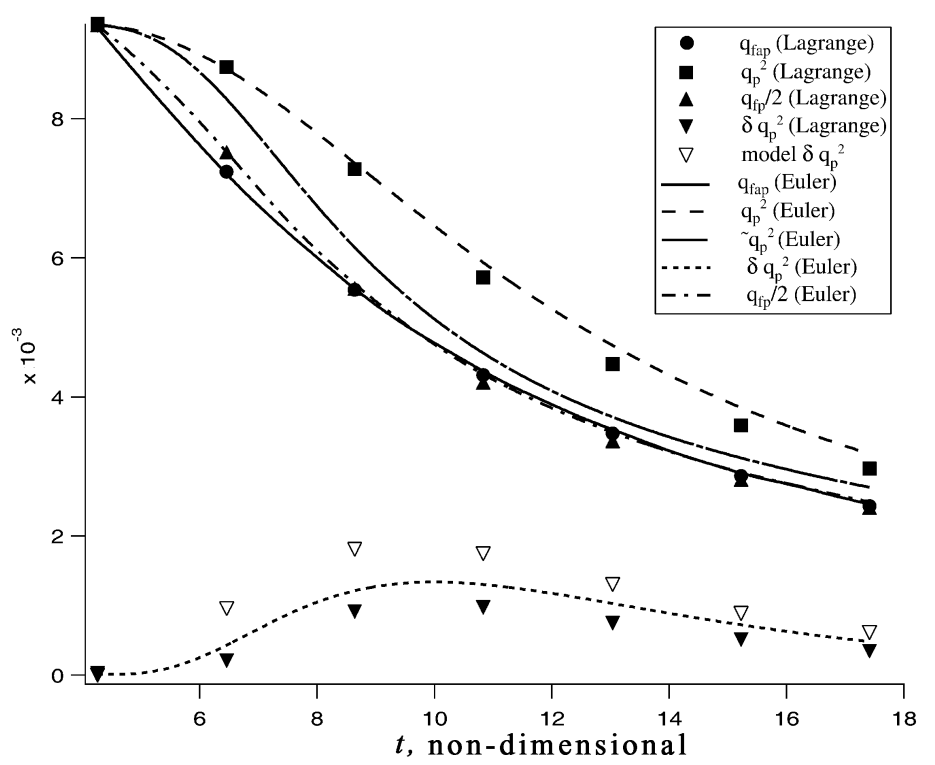

Fig. 2. Time evolution of the fluid and particle and fluid-particle velocity correlations in an homogeneous isotropic decaying two-phase turbulence; comparison of results from Lagrangian (symbols) and Eulerian (lines) simulations.

is zero since the particle velocity is identical to the carrier phase velocity, which are by definition correlated. This shows that the Eulerian simulation is able to capture the dynamics of the dispersed phase. In Fig. 3 the time scales from the Eulerian simulations are compared. Initially the relaxation time of the particles (solid line) is about half the dissipative time scale of the carrier phase (dashed line). This corresponds to the Stokes number of about 0.5. In the decreasing homogeneous isotropic turbulence, the dissipative time scale increases slowly and thus leads to a decreasing Stokes number (about 0.3 at the end of the Eulerian computation). The dissipative time scale of the fluid-particle correlation is initially close to the dissipative time scale of the carrier phase; it decreases in time so as to reach a level where it is about half the dissipative time scale of the carrier phase. This agrees with theoretical considerations $\left[{ }^{15}\right]$. Figure 4 shows snapshots of number density and RUM Energy in the Lagrangian and the Eulerian computation. The number density in the Eulerian computation admits smaller variations than in the Lagrangian computation due to the heuristically introduced bulk viscosity. This bulk viscosity acts on the compressible component of the velocity and thus limits compressibility effects. Therefore the particle number density is predicted to be more uniform than in the Lagrangian simulation. Random uncorrelated energy admits qualitatively the same structures in the Eulerian and Lagrangian computation. 


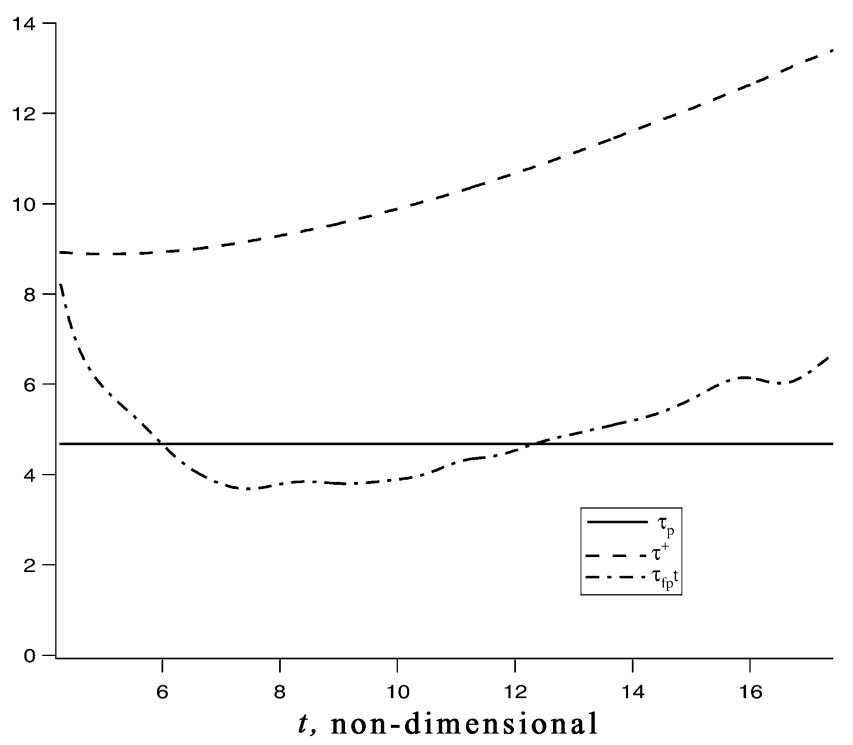

Fig. 3. Comparison of the dissipative time scale of the carrier phase $\tau^{+}$with the relaxation time of drag $\tau_{p}$ and the dissipative time scale of fluid-particle correlation $\tau_{f p}^{t}$.

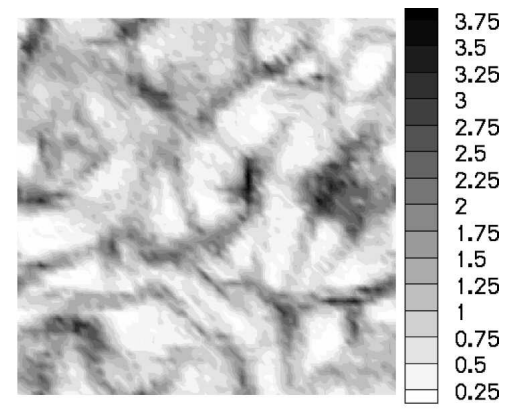

(a)

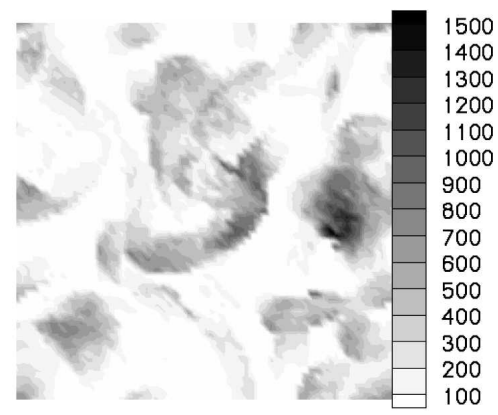

(c)

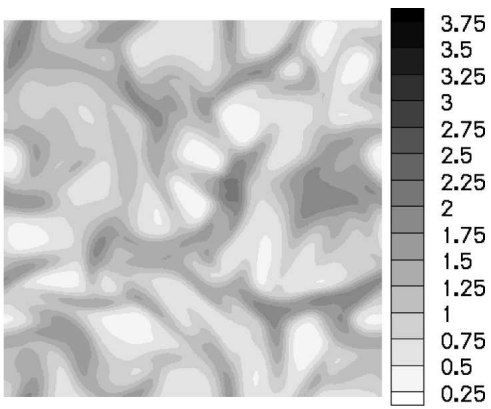

(b)

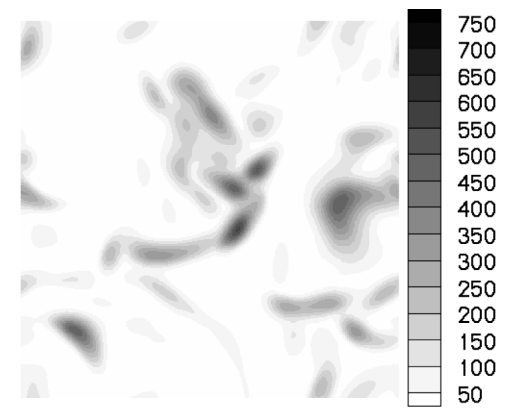

(d)

Fig. 4. Comparison of the normalized droplet number $\left(\tilde{n}_{p} /\left\langle\tilde{n}_{p}\right\rangle\right.$, a and b) and of the RUM Energy $\left(\delta \tilde{\theta}_{p}, \mathrm{c}\right.$ and $\mathrm{d}$ ) in the Lagrangian (a, c, resolution $\left.64^{3}\right)$ and the Eulerian (b, d, resolution $\left.128^{3}\right)$ computation after about one particle relaxation time $(t=10.8 \mathrm{~s})$ in the plane $z=0$. 
The heuristically introduced bulk viscosity term tends to render a more uniform spatial particle number distribution. Without this bulk viscosity term, calculations can currently not be carried out: particle segregation and enstrophy become too large. Since the spatial average of the volume viscosity is zero, it does not affect the temporal evolution of the averaged kinetic energy of RUM of the particles $\delta q_{p}^{2}$. Local instantaneous values of $\delta \tilde{\theta}_{p}$ may differ notably from the values obtained in the Lagrangian simulation. An important remaining question is therefore related to the subgrid model in the form of the bulk viscosity term. Higher spatial resolutions should clarify this problem if the encountered difficulties are related to numerical resolution. However, it is not clear if the modelling of stresses by Eq. (13) represents correctly the physics and if this is the origin of the encountered difficulties. This point is under current investigation with the strong support of Lagrangian simulations.

\section{CONCLUSION AND PROSPECT}

The presented study shows the capacity of Eulerian formalism to capture the dynamics of particles even in the vicinity of unity Stokes numbers. Simulations were performed at very small turbulent Reynolds numbers since simulations with higher Reynolds numbers of the carrier phase showed deficiencies in the spatial resolutions of the dispersed phase. Therefore tests have to be extended to higher Reynolds numbers and it would be interesting to develop a subgrid model for the dispersed phase. This would lead to large eddy simulations in an Eulerian framework which are very interesting for the unsteady computations of industrial applications with a high number of particles or droplets. Concerning dispersion measurements, it is interesting to extend the simulations by taking gravity into account and to test whether the crossing trajectory effect is captured in such an Eulerian framework.

\section{ACKNOWLEDGEMENTS}

Numerical computation of the Eulerian simulations were performed on the COMPAQ supercomputers of CEA and CERFACS. Numerical solutions on large grids $\left(192^{3}, 256^{3}\right)$ were performed on SGI ORIGIN 3800 at CINES in the framework of the EXTREME COMPUTING FOR TURBULENT COMBUSTION program using up to 128 processors. The Lagrangian reference solution was obtained with numerical simulations performed at the computing center IDRIS, using the Eulerian-Lagrangian version of NTMIX in the project Ecoulements Reactifs Diphasiques: Simulations Directes et aux Grandes Echelles.

Financial support for this work was received from the European Community via the STOPP research training network. 


\section{REFERENCES}

1. Simonin, O., Février, P. and Laviéville, J. On the spatial distribution of heavy particle velocities in turbulent flow: from continous field to particulate chaos. J. Turbulence, 2002, 3, 040, http://jot.iop.org/

2. Druzhinin, O. A. and Elghobashi, S. On the decay rate of isotropic turbulence laden with microparticles. Phys. Fluids, 1999, 11, 602-610.

3. Chapman, S. and Cowling, T. G. The Mathematical Theory of Non-Uniform Gases. Cambridge University Press, 1939.

4. Simonin, O. Combustion and turbulence in two phase flows. Lecture Series 1996-02, von Karman Institute for Fluid Dynamics, Rhode St. Genèse, Belgium, 1996.

5. Février, P., Simonin, O. and Squires, K. D. Partitioning of particle velocities in gassolid flows into a continuous field and a spatially-uncorrelated random distribution: theoretical formalism and numerical study. J. Fluid Mech. Forthcoming.

6. Reeks, M. W. On a kinetic equation fo the transport of particles in turbulent flows. Phys. Fluids A, 1991, 3, 446-456.

7. Février, P. Etude numerique des effets de concentration préférentielle et de corrélation spatiale entre vitesses des particules solides en turbulence homogène isotrope stationaire. PhD Thesis, INP, Toulouse, 2000.

8. Hirschfelder, J. O., Curtis, C. F. and Bird, R. B. Molecular Theory of Gases and Liquids. J. Wiley, 1954.

9. Elghobashi, S. and Truesdell, G. C. Direct simulation of particle dispersion in a decaying isotropic turbulence. J. Fluid Dynamics, 1992, 242, 655-700.

10. Snyder, W. H. and Lumley, J. L. Some measurements of particle velocity autocorrelation functions in a turbulent flow, Part 1. J. Fluid Mech., 1970, 48, 41-71.

11. Kaufmann, A., Simonin, O., Poinsot, T. and Helie, J. Dynamics and dispersion in Eulerian-Eulerian DNS of two-phase flows. In Proc. Summer Program 2002, Studying Turbulence Using Numerical Simulation Databases IX. Center for Turbulence Research, Stanford, Ca, 2002, 381-392.

12. Yeung, P. K. and Pope, S. B. An algorithm for tracking fluid particles in numerical simulations of homogeneous turbulence. J. Comput. Phys., 1988, 79, 373-416.

13. Chassing, P. Turbulence en mécanique des fluides. Collection POLYTECH, CEPADUES ed., 2000.

14. Laviéville, J. Simulations numériques et modelisation des interactions entre l'entrainement par la turbulence et les collisions interparticulaires en écoulements gaz-solides. PhD Thesis, Université de Rouen, 1997.

15. Simonin, O., Deutsch, E. and Bovin, M. Large eddy simulation and second-moment closure model of particle fluctuating motion in two-phase turbulent shear flows. In Selected Papers from the Ninth Symposium on Turbulent Shear Flows (Durst, F., Kasagi, N., Launder, B. E., Schmidt, F. W. and Whitelaw, J. H., eds.). Kyoto, Springer, 1993, 85-115. 


\section{Lagrange'i ja Euleri meetodite võrdlus osakeste arvmodelleerimisel homogeense isotroopse kõduva turbulentsi juhul}

André Kaufmann, Jerome Helie, Olivier Simonin ja Thierry Poinsot

On käsitletud otsese arvmodelleerimise lähendusmeetodit kahefaasilise dispersse Euler-Euleri vooluse juhul. On näidatud vajadust lülitada dispersse faasi momendi transpordivõrrandisse pingeliige ja esitatud lihtne mudel selle liikme määramiseks. Dispersset faasi iseloomustavate suuruste nagu osakeste arvulise tiheduse, makroskoopilise kiiruse ja pingetensori komponentide määramine võrdlusarvutustega, kasutades osakeste jälgimiseks Lagrange'i meetodit, kinnitab Euler-Euleri lähendusmeetodiga saadud tulemusi. 